\title{
Lévy Flights and Hydrodynamic Superdiffusion on the Dirac Cone of Graphene
}

\author{
Egor I. Kiselev ${ }^{1}$ and Jörg Schmalian ${ }^{1,2}$ \\ ${ }^{1}$ Institut für Theorie der Kondensierten Materie, Karlsruher Institut für Technologie, 76131 Karlsruhe, Germany \\ ${ }^{2}$ Institut für Festkörperphysik, Karlsruher Institut für Technologie, 76131 Karlsruhe, Germany
}

(Received 17 June 2019; published 8 November 2019)

\begin{abstract}
We show that the hydrodynamic collision processes of graphene electrons at the neutrality point can be described in terms of a Fokker-Planck equation with a fractional derivative, corresponding to a Lévy flight in momentum space. Thus, electron-electron collisions give rise to frequent small-angle scattering processes that are interrupted by rare large-angle events. The latter give rise to superdiffusive dynamics of collective excitations. We argue that such superdiffusive dynamics is of more general importance to the out-of-equilibrium dynamics of quantum-critical systems.
\end{abstract}

DOI: 10.1103/PhysRevLett.123.195302

The kinetics of large gravitational systems such as globular clusters in galaxies or of a classical charged plasma are governed by continuous collisions with small-angle scattering. The origin for this behavior is the long-range character of the Newton or Coulomb force, respectively. Such small-angle collisions behave in velocity space like drag and diffusion events, where a FokkerPlanck equation offers an efficient description [1-3]. Collisions can thus be seen as a Gaussian random walk in phase space. The velocity of a plasma or gravitational dust particle undergoes ordinary Brownian motion.

Quantum many-body systems that are near a quantumcritical point are governed by soft modes that will also induce effective long-range interactions [4]. This begs the question of whether such quantum-critical systems also allow for an effective Fokker-Planck description of the nonequilibrium kinetics, in the collision-dominated hydrodynamic regime and in the crossover regime from hydrodynamic to ballistic dynamics. Candidate systems are itinerant electrons near magnetic or nematic quantum phase transitions [5-14], the superconductor-insulator phase transition [15], or graphene near the Dirac point [16]. Anomalous diffusion was even shown to be present in two-dimensional Fermi liquids[17-23].

In this Letter, we analyze the quantum kinetics of graphene near the Dirac point with an electron-electron Coulomb interaction. We show that the kinetic theory at charge neutrality [24-28] can be expressed in terms of a Fokker-Planck equation, yet with a fractional derivative with respect to the momentum direction. The underlying random processes are Lévy flights [29-31], which are non-Gaussian random walks for which the step widths are distributed according to a power law. The slowly decaying tail of the step-width distribution makes it impossible to define a diffusion constant or to use a conventional Fokker-Planck equation. However, a diffusion equation of the form

$$
\frac{\partial \rho}{\partial t}+D_{\mu}|\triangle|^{\mu / 2} \rho=0
$$

with an appropriately generalized fractional derivative $[32,33]$ can be used to describe such random walks. Lévy flights have been discussed to model the migration pattern of animals as they search for resources [34,35], to model the high-frequency index dynamics of the stock market [36], or to describe distances between consecutive earthquakes [37]. In our system, they correspond to random walks in momentum space with power-law weight for large momentum-transfer processes. We demonstrate that the collision operator due to electron-electron interactions in graphene takes the form of a fractional derivative. Then, the Boltzmann equation becomes a fractional Fokker-Planck equation, which is similar to Eq. (1) with an exponent of $\mu=1$ :

$$
\left[\partial_{t}+\mathbf{v}_{\mathbf{k} \lambda} \cdot \nabla_{\mathbf{x}}-\tau_{L}^{-1}\left(\frac{\partial^{2}}{\partial \theta^{2}}\right)^{1 / 2}\right] f_{\mathbf{k} \lambda}=S_{\mathbf{k} \lambda},
$$

where $\theta$ determines the electron momentum direction: $\mathbf{k}=k(\cos \theta, \sin \theta)$. The precise definition of the fractional derivative is given below. This result implies that the outof-equilibrium dynamics of graphene in the hydrodynamic regime is governed by a wrapped Cauchy flight [38,39], which is a specific Lévy flight on the Dirac cone. In Fig. 1(a), we show a simulation of ordinary Brownian motion on a ring and of the wrapped Cauchy flight. Details of this simulation are summarized in the Supplemental Material [40], which includes Refs. [41-45]. The occurrence of rare large-angle jumps is clearly visible. The corresponding phase-space dynamics is sketched in Fig. 1(b). Although the direction of $\mathbf{k}$ undergoes anomalous diffusion, its magnitude $k \equiv|\mathbf{k}|$ is of the order of $k_{B} T / v_{0}$ with the graphene group velocity of $v_{0} \approx 10^{8} \mathrm{~cm} / \mathrm{s}$. The characteristic time of the process is $\tau_{L}$ with 


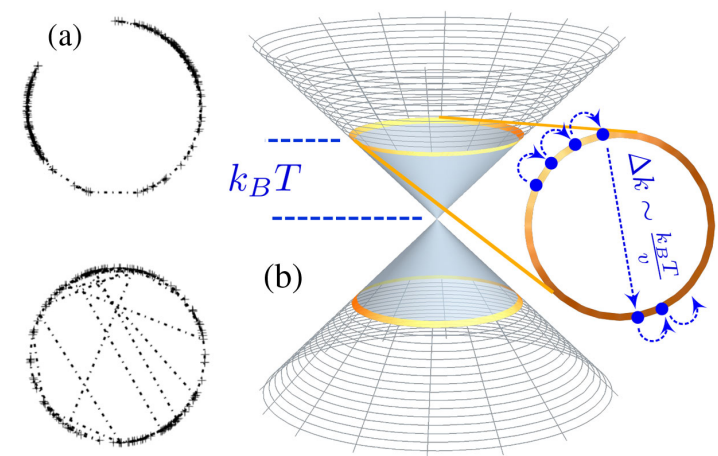

FIG. 1. (a) A wrapped Gaussian flight (upper circle) and a wrapped Cauchy flight (lower circle) with rare large momentumtransfer processes. (b) Illustration of Lévy flight in momentum space for graphene at the Dirac point. Electrons and holes that are thermally excited collide with each other. Most of the time, the momentum transfer due to the electron-electron Coulomb interaction leads to small-angle scattering. However, those processes are interrupted by rare processes with large momentum transfer. The latter change the dynamics of the system qualitatively, leading to an accelerated or superdiffusive dynamics.

$$
\hbar \tau_{L}^{-1} \approx 11.66 \alpha^{2} k_{B} T
$$

where the fine-structure constant of graphene is $\alpha=$ $e^{2} /\left(\hbar \epsilon v_{0}\right) \cdot \tau_{L}$ agrees up to a numerical coefficient with the collision time for the hydrodynamic transport behavior of graphene at the Dirac point [24-26]. Below, we discuss how $\tau_{L}$ is determined. Such a timescale was recently observed experimentally in the terahertz spectroscopy of graphene at charge neutrality [46].

Lévy flights in graphene have been discussed in Ref. [47], where an engineered distribution of adatoms was shown to result in a superdiffusive behavior of charge carriers, and in Ref. [48], in the context of highly photoexcited carriers that relax according to a cascade of processes - a behavior with interesting implications for pump-probe experiments. This can be seen as a superdiffusion in energy space far from equilibrium. It affects the magnitude of the momentum. Here, we focus on the low-energy hydrodynamic regime and find a very different behavior for the directional diffusion in momentum space. Nevertheless, these results strongly suggest that superdiffusive phase-space dynamics is a more common phenomenon in quantum-critical systems.

We start from the Boltzmann equation

$$
\left[\partial_{t}+\mathbf{v}_{\mathbf{k} \lambda} \cdot \nabla_{\mathbf{x}}+\mathbf{F}(\mathbf{x}, t) \cdot \nabla_{\mathbf{k}}+\mathcal{C}\right] f_{\mathbf{k} \lambda}(\mathbf{x}, t)=0
$$

for the electron distribution function $f_{\mathbf{k} \lambda}(\mathbf{x}, t)$, where $\mathbf{k}$ refers to the momentum and $\lambda= \pm 1$ labels the upper and lower cones of the Dirac spectrum $\varepsilon_{\mathbf{k} \lambda}=\lambda v_{0}|\mathbf{k}| . \mathbf{v}_{\mathbf{k} \lambda}=$ $\partial \varepsilon_{\mathbf{k} \lambda} / \partial \mathbf{k}$ is the velocity vector, and $\mathbf{F}(\mathbf{x}, t)$ is some external force, e.g., due to an external electric field. $\mathcal{C}$ is the Boltzmann collision operator due to electron-electron interactions and was derived to order $\alpha^{2}$ in Ref. [24] from a Keldysh-Schwinger approach; see this also in [40]. It takes the usual form of a two-body interaction:

$$
\begin{aligned}
\mathcal{C} f_{1}= & -\sum_{2,3,4} W_{12,34}\left[f_{1} f_{2}\left(1-f_{3}\right)\left(1-f_{4}\right) \mid\right. \\
& \left.-\mid\left(1-f_{1}\right)\left(1-f_{2}\right) f_{3} f_{4}\right] .
\end{aligned}
$$

The transition probability $W_{12,34}$ is due to the electronelectron Coulomb interaction $e^{2} / \epsilon$ of Dirac fermions that are confined to a two-dimensional system. $\epsilon$ is the dielectric constant determined by the substrate. For freestanding graphene, $\epsilon=1$ and the fine-structure constant $\alpha \approx 2.2$ is of order unity. A renormalization group analysis shows that $\alpha$ flows towards weak coupling, justifying our perturbative approach [16].

As usual, the kinetic distribution function $f_{\lambda, k}$ is expanded around the local equilibrium distribution $f_{k \lambda}^{0}=$ $\left(e^{\beta\left(\epsilon_{\lambda, k}-\mu\right)}+1\right)^{-1}$ and parametrized as $\left(f_{k}^{(0)}=f_{k+}^{(0)}\right)$ :

$$
f_{\mathbf{k} \lambda}(\mathbf{x}, t)=f_{k \lambda}^{(0)}+f_{k}^{(0)}\left(1-f_{k}^{(0)}\right) \psi_{\mathbf{k} \lambda}(\mathbf{x}, t) .
$$

We linearize the Boltzmann equation with respect to $\psi_{\mathbf{k} \lambda}(\mathbf{x}, t)$. With the Liouville operator

$$
\mathcal{L}=\left(\partial_{t}+\mathbf{v}_{\mathbf{k} \lambda} \cdot \nabla_{\mathbf{x}}\right) f_{k}^{(0)}\left(1-f_{k}^{(0)}\right),
$$

we obtain a compact formulation of the Boltzmann equation: $(\mathcal{L}+\mathcal{C}) \psi=S . S_{\mathbf{k} \lambda}(\mathbf{x}, t)$ contains external perturbations, such as those due to a space- and timedependent electric field or flow-velocity gradient. The operators $\mathcal{L}$ and $\mathcal{C}$ act on the momentum and band indices $\mathbf{k}$ and $\lambda$, respectively. Taking into account the kinematic constraints of the linear Dirac spectrum, the collision operator becomes

$$
\begin{aligned}
(\mathcal{C} \psi)_{\mathbf{k} \lambda}= & \frac{2 \pi}{\hbar} \int_{k^{\prime} q} \delta\left(k+k^{\prime}-|\mathbf{k}+\mathbf{q}|-\left|\mathbf{k}^{\prime}-\mathbf{q}\right|\right) \\
& \times\left(1-f_{k}^{(0)}\right)\left(1-f_{k^{\prime}}^{(0)}\right) f_{|\mathbf{k}+\mathbf{q}|}^{(0)} f_{\left|\mathbf{k}^{\prime}-\mathbf{q}\right|}^{(0)} \\
& \times\left\{\gamma_{\mathbf{k}, \mathbf{k}^{\prime}, \mathbf{q}}^{(1)}\left(\psi_{\mathbf{k}+\mathbf{q} \lambda}+\psi_{\mathbf{k}^{\prime}-\mathbf{q} \lambda}-\psi_{\mathbf{k}^{\prime} \lambda}-\psi_{\mathbf{k} \lambda}\right)\right. \\
& \left.+\gamma_{\mathbf{k}, \mathbf{k}^{\prime}, \mathbf{q}}^{(2)}\left(\psi_{\mathbf{k}+\mathbf{q} \lambda}-\psi_{-\mathbf{k}^{\prime}+\mathbf{q} \bar{\lambda}}+\psi_{-\mathbf{k}^{\prime} \bar{\lambda}}-\psi_{\mathbf{k} \lambda}\right)\right\},
\end{aligned}
$$

where the matrix elements $\gamma_{\mathbf{k}, \mathbf{k}^{\prime}, \mathbf{q}}^{(1,2)}$ are given in Ref. [40] and

$$
\int_{k} \cdots=\int \frac{d^{2} k}{(2 \pi)^{2}} \cdots
$$

One easily finds the zero modes that correspond to the conservation laws [24]. Equation (4) was obtained by projecting the distribution function onto the helical eigenstates of the problem. The same projection was 
performed in the derivation of the collision operator $[24,40]$.

The usual analysis of the Boltzmann equation proceeds as follows: One performs a Fourier transformation from $(\mathbf{x}, t)$ to $(\mathbf{q}, \omega)$ and introduces a complete set of states $\chi_{\mathbf{k} \lambda}^{(s)}$ to evaluate the matrix elements $\left\langle s|\mathcal{L}+\mathcal{C}| s^{\prime}\right\rangle$ with scalar product

$$
\left\langle s \mid s^{\prime}\right\rangle=\sum_{\mathbf{k} \lambda} \chi_{\mathbf{k} \lambda}^{(s) *} \chi_{\mathbf{k} \lambda}^{\left(s^{\prime}\right)}
$$

The Liouville operator becomes

$$
\mathcal{L}=\left(-i \omega+i \mathbf{v}_{\mathbf{k} \lambda} \cdot \mathbf{q}\right) f_{k}^{(0)}\left(1-f_{k}^{(0)}\right) .
$$

The distribution function then follows as $\psi=(\mathcal{L}+\mathcal{C})^{-1} S$. For finite $\omega$ or $\mathbf{q}$, the operator $\mathcal{L}+\mathcal{C}$ is nonsingular. This program is somewhat simplified for graphene at charge neutrality. As shown in Refs. [24-26,28], scattering processes where all momenta are collinear are enhanced by a factor $\log (1 / \alpha)$. This can be used to identify the dominant modes, which are derived in the Supplemental Material [40]:

$$
\chi_{\mathbf{k} \lambda}^{(m, s)}=\lambda^{m} e^{i m \theta}\left\{1, \lambda, \lambda v_{0} k /\left(k_{B} T\right)\right\},
$$

where $m \in \mathbb{Z}$ is the angular momentum quantum number, whereas $s \in\{1,2,3\}$ labels the collinear modes for given $m$. We solve the kinetic equation by projecting it onto the dominant collinear modes $\chi_{\mathbf{k} \lambda}^{(m, s)}$, but we checked that our key conclusions are unchanged if we choose a larger set of basis functions. Also, if we restrict our considerations to the transport of charge due to external electric fields, it suffices to consider the modes $\chi_{\mathbf{k} \lambda}^{(m, 1)}=\lambda^{m} e^{i m \theta}$ of Eq. (9). For simplicity, we confine ourselves to electric-field source terms and only discuss this mode. The generalization to other modes is straightforward.

The low-energy Dirac Hamiltonian is rotationally invariant such that the collision operator becomes diagonal in the angular momentum representation:

$$
\left\langle m|\mathcal{C}| m^{\prime}\right\rangle=\frac{\ln 2}{\pi} \delta_{m, m^{\prime}} \tau_{m}^{-1}
$$

The diagonal elements are, besides a convenient prefactor, the scattering rates of the corresponding angular momentum channel. $\tau_{0}^{-1}=0$ due to charge conservation, whereas the collision rate

$$
\hbar \tau_{1}^{-1}=3.646 \alpha^{2} k_{B} T
$$

for $m=1$ was determined in Ref. [24] to yield the optical conductivity
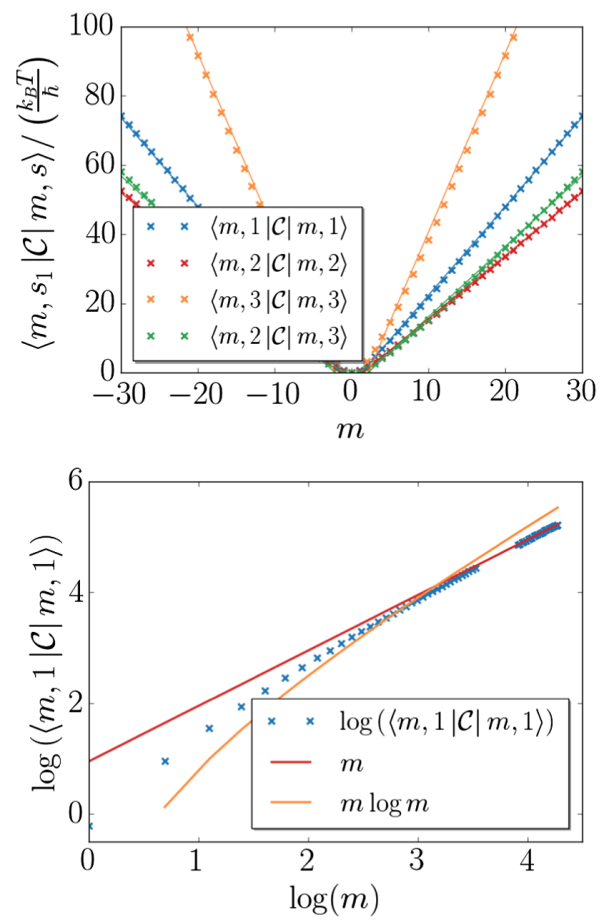

FIG. 2. Upper panel: Angular momentum dependence of matrix elements of the collision operator $\left\langle m, s|\mathcal{C}| m, s^{\prime}\right\rangle$, where $s \in$ $\{1,2,3\}$ refers to the collinear eigenmodes of Eq. (9). In the text, we discuss (for simplicity) only $\langle m|\mathcal{C}| m\rangle \equiv\langle m, 1|\mathcal{C}| m, 1\rangle$. Lower panel: log-log plot of matrix element demonstrating that we can distinguish the $|m|$ dependence from, e.g., $|m| \log |m|$.

$$
\sigma(\omega)=\frac{e^{2}}{h} 4 \ln 2 k_{B} T\left(-i \hbar \omega+\hbar \tau_{1}^{-1}\right)^{-1} .
$$

$\tau_{1}^{-1}$ was recently observed in Ref. [46] using a waveguide setup, which is a demonstration of quantum-critical hydrodynamic transport. The dramatic violation of the Wiedemann-Franz law at charge neutrality is another important indication for electronic hydrodynamics at charge neutrality [49].

We evaluated the matrix elements $\langle m|\mathcal{C}| m\rangle$ and obtain

$$
\tau_{m}^{-1}=\tau_{1}^{-1}\left(\kappa|m|-\kappa^{\prime}\right),
$$

where the two numerical constants are given as $\kappa \approx 3.199$ and $\kappa^{\prime} \approx 4.296$; see also Fig. 2. This behavior is asymptotically exact at large $m$ but already valid with good accuracy for $m>2$. The most important aspect of this result is that the dependence of the scattering rate on the angular momentum $m$ is nonanalytic. To simplify the analysis, we assume in the following that $\tau_{m}^{-1}=\tau_{L}^{-1}|m|$, where $\tau_{L}^{-1}=\kappa \tau_{1}^{-1}$ is the characteristic time of the Lévy flight process, which is given in Eq. (3).

The implication of the $|m|$ dependence of $\tau_{m}^{-1}$ becomes evident if we consider the scattering between two distinct momentum directions. Fourier transformation of $\tau_{m}^{-1}$ yields 


$$
\left\langle\theta|\mathcal{C}| \theta^{\prime}\right\rangle=-\frac{\ln 2 \tau_{L}^{-1}}{(2 \pi)^{2} \sin ^{2}\left[\left(\theta-\theta^{\prime}\right) / 2\right]}
$$

Thus, we obtain a slowly decaying power law of $\sim\left(\theta-\theta^{\prime}\right)^{-2}$ for scattering processes away from forward scattering. Using this result for $\left\langle\theta|\mathcal{C}| \theta^{\prime}\right\rangle$, we can rewrite the Boltzmann equation in the form of Eq. (2) with the characteristic time $\tau_{L}$ of Eq. (3) for the Lévy flight. To arrive at Eq. (2), we used that the convolution of the distribution function with $\left\langle\theta|\mathcal{C}| \theta^{\prime}\right\rangle$ can be expressed as a fractional derivative

$$
\left(\frac{\partial^{2} g(\theta)}{\partial \theta^{2}}\right)^{1 / 2}=\frac{\partial}{\partial \theta} \int_{0}^{2 \pi} \frac{g\left(\theta^{\prime}\right)}{\tan \left[\left(\theta-\theta^{\prime}\right) / 2\right]} d \theta^{\prime}
$$

Eq. (14) is a special case of the Riesz-Feller derivative $\triangle^{\mu / 2}[32,33]$.

There are some profound implications that this fractional Fokker-Planck formulation immediately reveals. For example, we consider a scenario where we inject a highly directed excitation [23]. To this end, we consider a source term in the Boltzmann equation that causes this excitation:

$$
S_{\mathbf{k} \lambda}(t)=\delta(t) f_{k}^{(0)}\left(1-f_{k}^{(0)}\right) \sum_{m} \delta h_{\lambda m} e^{i m \theta} .
$$

We assume that we will only inject excitations in a window $\pm k_{B} T$ near the Dirac point, hence the factor $f_{k}^{(0)}\left(1-f_{k}^{(0)}\right)$. In addition, we decompose the source term into its angular momentum modes. The linearized Boltzmann equation is applicable if $\left|\delta h_{\lambda m}\right| \ll 1$. To describe an excitation that is peaked along an axis given by a certain momentum direction, we use $\delta h_{\lambda, m}=\delta h \lambda^{m}$, which has the same $\lambda$ dependence as the $s=1$ mode of Eq. (9). The solution of the fractional Fokker-Planck equation for a homogeneous case of $\mathbf{q}=0$ is then given as

$$
\psi_{\lambda}(\theta, t)=\delta h \Theta(t) \frac{\sinh \left(t / \tau_{L}\right)}{\cosh \left(t / \tau_{L}\right)-\lambda \cos (\theta)} .
$$

This function is known as a wrapped Cauchy distribution with circular variance $1-e^{-t / \tau_{L}}[38,39] . \Theta(t)$ is the step function. $\psi_{+}(\theta, t)$ is shown in the upper panel of Fig. 3.

For $t=0, \psi_{\lambda}(\theta, t)$ corresponds to two delta functions due to particle and hole flows in opposite directions. Let us concentrate on the particle channel $\lambda=+1$. For short times of $t \ll \tau_{L}$, the peak in the initial current direction decays as

$$
\psi_{+}(t, \theta=0) \approx \delta h \frac{\tau_{L}}{\pi t},
$$

whereas the distribution function grows linearly for all nonzero angles:
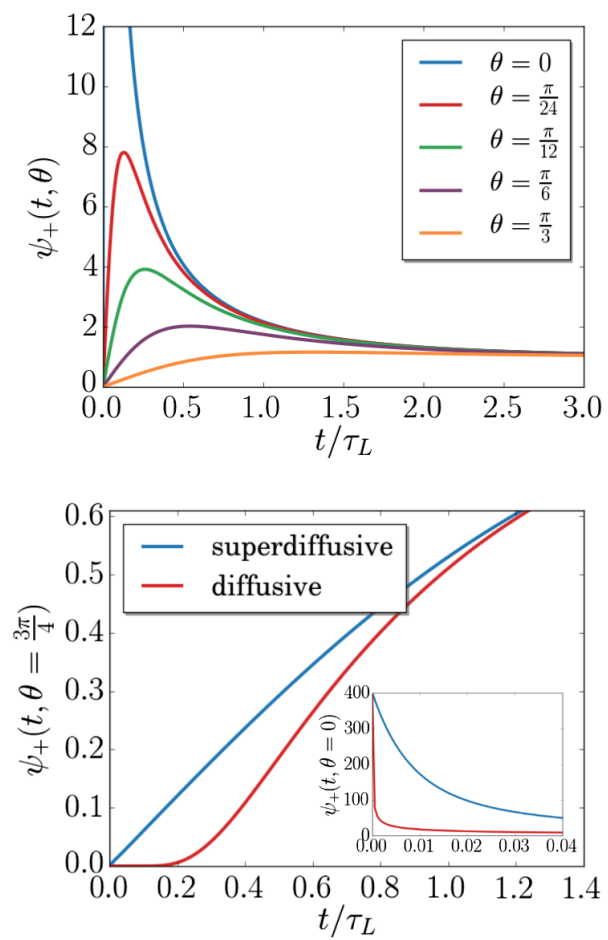

FIG. 3. Upper panel: Postinjection distribution function that follows from the fractional Fokker-Planck equation [Eq. (2)] with the external perturbation of Eq. (15). Notice the superdiffusive dynamics at short times. Lower panel: Comparison of superdiffusive and diffusive dynamics at short times. At angles away from the peak at $\theta=0$, superdiffusion leads to a faster growth of the distribution function. Inset: The initial peak at $\theta=0$ decays as $1 / t$ for superdiffusion and $1 / \sqrt{t}$ for ordinary diffusion. This behavior dominates the heating of the system (see main text).

$$
\psi_{+}(t, \theta \neq 0) \approx \frac{\delta h}{4 \pi \sin ^{2}(\theta / 2)} \frac{t}{\tau_{L}} .
$$

The same behavior occurs for $\lambda=-1$ if we shift $\theta \rightarrow \theta+\pi$. This behavior is in contrast to the one that follows from usual Fokker-Planck diffusion. The latter we obtain, for example, from collision rates $\tau_{m}^{-1} \sim m^{2}$. Then, the usual spreading of a Gaussian wave package occurs with $\psi_{+}(t, \theta=0) \propto t^{-1 / 2}$ and $\psi_{+}(t, \theta \neq 0) \propto t^{2}$ (lower panel of Fig. 3). Although the forward direction of the distribution decays more slowly for Lévy flights than in usual diffusion, the growth at larger angles is much faster, hence the name superdiffusion.

A tangible implication of this superdiffusive charge motion is the heating of the system after the injection. To this end, we determine the time dependence of the entropy density

$$
\frac{\partial s(t)}{\partial t}=4 k_{B} \sum_{\lambda} \int_{k} \log \left(\frac{1-f_{\mathbf{k} \lambda}}{f_{\mathbf{k} \lambda}}\right) \frac{\partial f_{\mathbf{k} \lambda}}{\partial t} .
$$

The heat density caused by the injection is given by $\delta q(t)=T\left[s_{\mathrm{eq}}-s(t)\right]$. Inserting the distribution function of Eq. (16), we obtain 


$$
\frac{\partial s(t)}{\partial t}=\frac{4 \log 2}{9 \zeta(3)} \frac{s_{\mathrm{eq}}}{\tau_{L}} \frac{(\delta h)^{2}}{\sinh ^{2}\left(t / \tau_{L}\right)}
$$

where $s_{\mathrm{eq}}$ is the equilibrium entropy density. In order to stay within the regime of linear response, we are confined to $t>\delta h \tau_{L}$. For $t \rightarrow \infty$, one finds $s \rightarrow s_{\text {eq }}$; and we obtain

$$
s(t)=s_{\mathrm{eq}}\left(1-(\delta h)^{2} \frac{4 \log (2)}{9 \zeta(3)}\left[\operatorname{coth}\left(\frac{t}{\tau_{L}}\right)-1\right]\right) .
$$

Thus, initial heating occurs according to

$$
\delta q(t) \propto T s_{\mathrm{eq}} \delta h^{2} \frac{\tau_{L}}{t} .
$$

This result is a direct consequence of the superdiffusive behavior: in particular, of the slow decay along the forward direction. In the case of ordinary diffusion follows instead of Eq. (21) $\delta q(t) \propto t^{-1 / 2}$, which is a faster behavior then that of Eq. (21) (see Fig. 3 and [40]). However, adjusting $\delta h$ such that the same amount of heat is produced for Lévy and diffusive behaviors, the initial heating of the Lévy flight is larger. The $m$ dependence of $\tau_{m}^{-1}$ that is responsible for the Lévy flight behavior can also be seen in nonlocal transport coefficients because the conductivity at finite momentum $\mathbf{q}$ couples the different harmonics of the distribution function. As an example, we show, in the Supplemental Material [40], the transverse optical conductivity at finite $\mathbf{q}$. Nevertheless, experiments with directed electron beams [50], which in the past have been used to investigate electron-electron scattering effects [51], seem to offer a more direct way of testing the short time behavior of Eq. (21).

The occurrence of Lévy flights to describe scattering processes in momentum space is a more general phenomenon and not restricted to graphene at the neutrality point. In two-dimensional Fermi liquids with characteristic rate $\hbar \tau_{\mathrm{FL}}^{-1} \sim k_{B} T^{2} / T_{F}$, it holds for $|m|<M \sim \sqrt{T_{F} / T}$ that $\tau_{m}{ }^{-1} \sim \tau_{\mathrm{FL}}^{-1}\left(m^{p} / M^{p}\right) \log |m| \quad$ with $\quad p=2\left[1+(-1)^{m}\right]$, whereas $\tau_{m}^{-1} \sim \tau_{\mathrm{FL}}^{-1}$ for $|m|>M[18,23] . T_{F}$ is the Fermi temperature. This yields superdiffusive behavior in a wide time window. Another system that also shows $\tau_{m}^{-1} \propto|m|$ for arbitrarily large $m$ consists of electrons in a random magnetic field, which are important for the description of composite fermions in the fractional quantum Hall regime [52]. Our analysis implies that this system should also undergo a wrapped Cauchy flight in momentum space. Large classes of quantum-critical systems (discussed, e.g., in Refs. [5-15]) are governed by long-ranged soft-mode interactions. An analysis of collision processes along the lines discussed here may reveal a nonanalytic dependence of the scattering rates on an angular momentum quantum number according to $\tau_{m}^{-1} \propto|m|^{\mu / 2}$. This would give rise to a more general class of wrapped Lévy flights, which are a consequence of the power-law behavior $\left\langle\theta|\mathcal{C}| \theta^{\prime}\right\rangle \propto$ $\left|\theta-\theta^{\prime}\right|^{-1-(\mu / 2)}$ near forward scattering. This could occur on the Fermi surface for itinerant quantum-critical systems or near a soft momentum in critical bosonic systems. If a fractional Fokker-Planck formulation, along the lines of our Eq. (2), can be derived, it will be significantly easier to draw conclusions about the out-of-equilibrium dynamics of the system, such as a focussed injection of collective excitations. Finally, we mention that the formulation of the Boltzmann equation presented here can also be used to study the nonlocal electric and thermal conductivities and viscosities, allowing insight into the diffusive and sound excitations in the hydrodynamic regime [53].

We are grateful to Andrey V. Chubukov, Leonid S. Levitov, Dimitrii L. Maslov, and Alexander D. Mirlin for stimulating discussions; and to the European Commission's Horizon 2020 RISE program Hydrotronics (Grant No. 873028) for support.

[1] S. Chandrasekhar, Stochastic problems in physics and astronomy, Rev. Mod. Phys. 15, 1 (1943).

[2] M. N. Rosenbluth, W. M. MacDonald, and D. L. Judd, Fokker-Planck equation for an inverse-square force, Phys. Rev. 107, 1 (1957).

[3] D. F. Chernoff and M. D. Weinberg, Evolution of globular clusters in the Galaxy, Astrophys. J. 351, 121 (1990).

[4] S. Sachdev, Quantum Phase Transitions (Cambridge University Press, Cambridge, England, 1999).

[5] J. A. Hertz, Quantum critical phenomena, Phys. Rev. B 14, 1165 (1976).

[6] T. Moriya, Spin Fluctuations in Itinerant Electron Magnetism, Springer Series in Solid-State Science Vol. 52 (Springer, Berlin, 1984).

[7] A. J. Millis, Effect of a nonzero temperature on quantum critical points in itinerant fermion systems, Phys. Rev. B 48, 7183 (1993).

[8] R. B. Laughlin, G. G. Lonzarich, P. Monthoux, and D. Pines, The quantum criticality conundrum, Adv. Phys. 50, 361 (2001).

[9] A. J. Millis, A. J. Schofield, G. G. Lonzarich, and S. A. Grigera, Metamagnetic Quantum Criticality, Phys. Rev. Lett. 88, 217204 (2002).

[10] Ar. Abanov, A. V. Chubukov, and J. Schmalian, Quantumcritical theory of the spin-fermion model and its application to cuprates: Normal state analysis, Adv. Phys. 52, 119 (2003).

[11] H. Löhneysen, A. Rosch, M. Vojta, and P. Wölfle, Fermiliquid instabilities at magnetic quantum phase transitions, Rev. Mod. Phys. 79, 1015 (2007).

[12] L. Dell'Anna and W. Metzner, Electrical Resistivity Near Pomeranchuk Instability in Two Dimensions, Phys. Rev. Lett. 98, 136402 (2007).

[13] M. A. Metlitski and S. Sachdev, Quantum phase transitions of metals in two spatial dimensions. I. Ising-nematic order, Phys. Rev. B 82, 075127 (2010).

[14] Y. Schattner, S. Lederer, S. A. Kivelson, and E. Berg, Ising Nematic Quantum Critical Point in a Metal: A Monte Carlo Study, Phys. Rev. X 6, 031028 (2016). 
[15] K. Damle and S. Sachdev, Nonzero-temperature transport near quantum critical points, Phys. Rev. B 56, 8714 (1997).

[16] Daniel E. Sheehy and Jörg Schmalian, Quantum Critical Scaling in Graphene, Phys. Rev. Lett. 99, 226803 (2007).

[17] R. N. Gurzhi, A. N. Kalinenko, and A. I. Kopeliovich, Electron-Electron Collisions and a New Hydrodynamic Effect in Two-Dimensional Electron Gas, Phys. Rev. Lett. 74, 3872 (1995).

[18] R. N. Gurzhi, A. N. Kalinenko, and A. I. Kopeliovich, Inefficiency of odd relaxation and propagation of electron beams in a two-dimensional electron system, Surf. Sci. 361-362, 497 (1996).

[19] D. L. Maslov, V. I. Yudson, and A. V. Chubukov, Resistivity of a Non-Galilean-Invariant Fermi Liquid near Pomeranchuk Quantum Criticality, Phys. Rev. Lett. 106, 106403 (2011).

[20] H. K. Pal, V. I. Yudson, and D. L. Maslov, Resistivity of non-Galileian invariant Fermi- and non-Fermi liquids, Lith. J. Phys. 52, 142 (2012).

[21] D. L. Maslov and A. V. Chubukov, Optical response of correlated electron systems, Rep. Prog. Phys. 80, 026503 (2017).

[22] P. Ledwith, H. Guo, A. Shytov, and L. Levitov, Tomographic Dynamics and Scale-Dependent Viscosity in 2D Electron Systems, Phys. Rev. Lett. 123, 116601 (2019).

[23] P. J. Ledwith, H. Guo, and L. Levitov, The hierarchy of excitation lifetimes in two-dimensional Fermi gases, Ann. Phys. (Amsterdam), 411, 167913 (2019).

[24] L. Fritz, J. Schmalian, M. Müller, and S. Sachdev, Quantum critical transport in clean graphene, Phys. Rev. B 78, 085416 (2008).

[25] A. B. Kashuba, Conductivity of defectless graphene, Phys. Rev. B 78, 085415 (2008).

[26] M. Müller, J. Schmalian, and L. Fritz, Graphene: A Nearly Perfect Fluid, Phys. Rev. Lett. 103, 025301 (2009).

[27] M. Schütt, P. M. Ostrovsky, I. V. Gornyi, and A. D. Mirlin, Coulomb interaction in graphene: Relaxation rates and transport, Phys. Rev. B 83, 155441 (2011).

[28] E. I. Kiselev and J. Schmalian, Boundary conditions of viscous electron flow, Phys. Rev. B 99, 035430 (2019).

[29] P. Lévy, Théorie de l'Addition des Variables Aléatoires (Gauthier-Villars, Paris, 1954).

[30] B. Mandelbrot, The Fractal Geometry of Nature (Freeman, New York, 1977).

[31] W. Feller, An Introduction to Probability Theory and its Applications (Wiley, New York, 1971), 2nd ed., Vol. 2.

[32] S. G. Samko, A. A. Kilbas, and O. I. Marichev, Fractional Integrals and Derivatives, Theory and Applications (Gordon and Breach, Amsterdam, 1993).

[33] R. Hermann, Fractional Calculus: An Introduction for Physicists, 2nd ed. (World Scientific, New Jersey, 2014).

[34] F. Bartumeus, M. G. E. Da Luz, G. M. Viswanathan, and J. Catalan, Animal search strategies: A quantitative randomwalk analysis, Ecology 86, 3078 (2005).

[35] A. M. Reynolds and C. J. Rhodes, The Lévy flight paradigm: Random search patterns and mechanisms, Ecology 90, 877 (2009).

[36] R. N. Mantegna and H. E. Stanley, Econophysics: Scaling and its breakdown in finance, J. Stat. Phys. 89, 469 (1997).
[37] A. Corral, Universal Earthquake-Occurrence Jumps, Correlations with Time, and Anomalous Diffusion, Phys. Rev. Lett. 97, 178501 (2006).

[38] P. Levy, L'addition des variables aléatoires définies sur une circonférence, Bull. Soc. Math. Fr. 67, 1 (1939).

[39] K. V. Mardia and P. E. Jupp, Directional Statistics (Wiley, New York, 1999), ISBN 978-0-471-95333-3.

[40] See Supplemental Material at http://link.aps.org/ supplemental/10.1103/PhysRevLett.123.195302 for a description of the simulations leading to Fig. 1(a), a derivation of the collinear zero modes of Eq. (9), a detailed discussion of the Boltzmann collision operator, and a discussion of the dependence of the non-local conductivity on the relaxation times of Eq. (12) .

[41] A. V. Chechkin, V. Y. Gonchar, J. Klafter, R. Metzler, and L. V. Tanatarov, Lévy flights in a steep potential well, J. Stat. Phys. 115, 1505 (2004).

[42] R. Metzler, A. V. Chechkin, and J. Klafter, Levy Statistics and Anomalous Transport: Levy Flights and Subdiffusion, Encyclopedia of Complexity and Systems Science (Springer, New York, 2012), p. 5218.

[43] G. Kurz, I. Gilitschenski, and U. D. Hanebeck, in 2014 Sensor Data Fusion: Trends, Solutions, Applications (SDF) (IEEE, New York, 2014).

[44] A. Wixforth, J. P. Kotthaus, and G. Weimann, Quantum Oscillations in the Surface-Acoustic-Wave Attenuation Caused by a Two-Dimensional Electron System, Phys. Rev. Lett. 56, 2104 (1986).

[45] S. H. Simon, Coupling of surface acoustic waves to a twodimensional electron gas, Phys. Rev. B 54, 13878 (1996).

[46] P. Gallagher, C.-S. Yang, T. Lyu, F. Tian, R. Kou, H. Zhang, K. Watanabe, T. Taniguchi, and F. Wang, Quantum-critical conductivity of the Dirac fluid in graphene, Science 364, 158 (2019).

[47] S. Gattenlöhner, I. V. Gornyi, P. M. Ostrovsky, B. Trauzettel, A. D. Mirlin, and M. Titov, Lévy Flights Due to Anisotropic Disorder in Graphene, Phys. Rev. Lett. 117, 046603 (2016).

[48] U. Briskot, I. A. Dmitriev, and A. D. Mirlin, Relaxation of optically excited carriers in graphene: Anomalous diffusion and Lévy flights, Phys. Rev. B 89, 075414 (2014).

[49] J. Crossno, J. K. Shi, K. Wang, X. Liu, A. Harzheim, A. Lucas, S. Sachdev, P. Kim, T. Taniguchi, K. Watanabe, T. A. Ohki, and K. C. Fong, Observation of the Dirac fluid and the breakdown of the Wiedemann-Franz law in graphene, Science 351, 1058 (2016).

[50] K. Wang, M. M. Elahi, L. Wang, K. M. M. Habib, T. Taniguchi, K. Watanabe, J. Hone, A. W. Ghosh, G.-H. Lee, and P. Kim, Graphene transistor based on tunable Dirac fermion optics, Proc. Natl. Acad. Sci. U.S.A. 116, 6575 (2019).

[51] H. Predel, H. Buhmann, L. W. Molenkamp, R. N. Gurzhi, A. N. Kalinenko, A. I. Kopeliovich, and A. V. Yanovsky, Effects of electron-electron scattering on electron-beam propagation in a two-dimensional electron gas, Phys. Rev. B 62, 2057 (2000).

[52] A. D. Mirlin and P. Wölfle, Composite Fermions in the Fractional Quantum Hall Effect: Transport at Finite Wave Vector, Phys. Rev. Lett. 78, 3717 (1997).

[53] E. I. Kiselev and J. Schmalian (unpublished). 